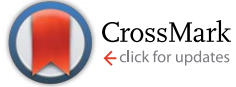

Cite this: RSC Adv., 2016, 6, 112520
Received 30th September 2016 Accepted 21st November 2016

DOI: $10.1039 / c 6 r a 24412 b$

www.rsc.org/advances

\section{Controlling luminescent silicon nanoparticle emission produced by nanosecond pulsed laser ablation: role of interface defect states and crystallinity phase}

\begin{abstract}
T. G. Ulusoy Ghobadi,,$^{\text {ab }}$ A. Ghobadi, tbc T. Okyay, ${ }^{d}$ K. Topalliab and A. K. Okyay $\AA^{\star a b c}$
In this study, we provide a systematic study on the origin of green and blue emission from luminescent silicon nanoparticles (Si-NPs) synthesized in water using a nanosecond pulsed laser ablation methodology. Here we report a direct one-step process to make ultra-small Si-NPs ( $3 \mathrm{~nm}$ mean size) by utilizing spiral beam scanning. In each ablation cycle, this scanning scheme collects generated nanoparticles towards the center and ablates them in subsequent cycles. Therefore, the resultant Si-NPs can reach very small sizes in a short time with high uniformity in their size distribution. Further, we investigate the effect of laser fluence on the emission properties of the obtained nanoparticles. For this aim, two different values of $60 \mathrm{~mJ} \mathrm{~cm} \mathrm{~cm}^{-2}$ and $30 \mathrm{~mJ} \mathrm{~cm}$-2 laser fluences are employed to achieve green and blue emitting Si-NPs, respectively. Our results show that the emission spectrum for both blue and green Si-NPs has two main peaks at $426 \mathrm{~nm}$ and $520 \mathrm{~nm}$, but their relative intensity ratios are different for these two cases. The findings of this study suggest that the blue emission originates from oxide related surface defects at the $\mathrm{Si} / \mathrm{SiO}_{x}$ interface, while the green emission stems from grain boundaries existing at the NP surface. We found that these two types of disorders can be controlled by tuning the laser power.
\end{abstract}

\section{Introduction}

Silicon, as the core of semiconductor technology, has been extensively employed in a variety of applications spanning from energy and environmental sciences to biomedical and biotechnologies. However, its inherent indirect band gap properties have limited its use in photovoltaic and light emitting diode applications. When the dimensions of Si nanoparticles (NPs) become comparable or smaller than Bohr radius of a bulk exciton $(\sim 4 \mathrm{~nm} \text { for Si) })^{1-4}$ they undergo a remarkable transition from indirect to direct band gap semiconductor. ${ }^{5}$ Under such conditions, band-to-band radiative recombination (rather than phonon-assisted indirect transitions) begins to dominate Si-NPs' luminescence properties. The efficient luminescence of ultra-small silicon nanoparticle (Si-NPs) $(<\sim 4 \mathrm{~nm}$ exciton Bohr radius of $\mathrm{Si}$ ) opened myriad of possibilities in

\footnotetext{
${ }^{a}$ UNAM - National Nanotechnology Research Center, Bilkent University, 06800 Ankara, Turkey.E-mail: aokyay@stanfordalumni.org

${ }^{b}$ Institute of Materials Science and Nanotechnology, Bilkent University, 06800 Ankara, Turkey

'Department of Electrical and Electronics Engineering, Bilkent University, 06800 Ankara, Turkey

${ }^{d}$ OkyayTech R\&D Ltd, Cyberpark, Bilkent University, 06800 Ankara, Turkey

\$ These authors contributed equally to this work.

$\dagger$ Dr Okyay was with Bilkent University at the time of the study.
}

application areas such as solid state lighting, ${ }^{6}$ full color displays, ${ }^{7,8}$ optical sensors ${ }^{9}$ and fluorescent tags for biological imaging. ${ }^{10}$

Up to now, several chemical and physical methods have been developed for the Si-NPs synthesis, including solution phase synthesis by reducing silicon halides, ${ }^{\mathbf{1 1}-14}$ electrochemical etching of a silicon wafer with a mixed solution of aqueous $\mathrm{HF}, \mathrm{H}_{2} \mathrm{O}_{2}$ and methanol, ${ }^{15,16}$ RF plasma decomposition of silane precursors $\left(\mathrm{SiH}_{4}\right),{ }^{17,18}$ and $\mathrm{CO}_{2}$ laser pyrolysis of silane. ${ }^{19,20}$ Although, chemical methods offer high control on particle size and surface properties but they usually have more complicated synthesis routes and several chemical precursors and reducing agents are involved during these processes. Thus each method requires optimization of chemical products, reducing agents or many steps for well controlled NP size. In recent years, pulsed laser ablation in liquid gathered immense interest as an efficient and alternative technique since it offers several advantages which can be listed as follows: (1) no material restriction compared to other techniques (2) no requirement for chemical precursors or reactions (3) no toxic by-products. However, the main drawback of this method is the limited control on the resulting size of the nanoparticles and their size distribution (homogeneity). To tackle with this problem, colloidal $\mathrm{Si}$ nanoparticles production has been studied employing different laser types with different 
wavelengths and pulse durations..$^{21,22}$ In some of these studies, nanosecond laser pulses have been utilized which results in the formation of wide size distribution of nanoparticles which stabilize in the form of clusters. ${ }^{23,24}$ Therefore, some posttreatments are necessary to avoid agglomeration and enlarging of nanoparticles. ${ }^{25}$ This issue is less destructive for femtosecond laser in which a better size control mechanism can be obtained by tuning the laser pulse energy. On the other hand, several studies have been conducted to understand the essence of Si-NPs emission.

Several mechanisms have been proposed to explain emission properties of NPs..$^{26-28}$ The main ones are quantum confinement effects, oxide related defects and combination of these two phenomena. However, its origin is still controversial and it highly depends on NPs preparation route. In present work, we propose a facile and widely adopted way to synthesize highly uniform sub-5 $\mathrm{nm}$ nanoparticles by combining pulsed laser ablation technique with spiral shaped laser beam scanning. In this type of scanning, the particles are collected towards the center of the spiral as they are produced and therefore reablated continuously in every cycle. Consequently, highly uniform ultra-small nanoparticles (with dimensions as small as $\sim 3 \mathrm{~nm}$ ) can be achieved. On the other side, we performed this ablation specifically on two different levels of laser fluence (30 $\mathrm{mJ} \mathrm{cm}{ }^{-2}$ and $60 \mathrm{~mJ} \mathrm{~cm}^{-2}$ ) to obtain highly uniform green and blue luminescent Si-NPs. A systematic study has been conducted to get a detailed analysis on the origin of these blue and green emissions. Our findings reveal that by adjusting laser parameters and scanning scheme, highly uniform Si-NPs with tunable emission can be obtained using nanosecond pulsed laser ablation.

\section{Experimental}

In brief, the laser system used in this study is comprised of a Nufern NuQ fiber laser (NUQA-1064-NA-0030-F1) operated at ambient temperature with $1064 \mathrm{~nm}$ wavelength, $100 \mathrm{~ns}$ pulse width duration, a repetition rate/frequency of $30 \mathrm{kHz}$ pulse repetition rate and uses a pulse energy of $1 \mathrm{~mJ}$. To synthesize the silicon colloidal nanoparticles, $500 \mu \mathrm{m}$-thick p-type silicon wafer ( $\langle 100\rangle$ direction, resistivity $0.1 \mathrm{ohm}-\mathrm{cm}$, purity $99.999 \%)$ is used as a bulk target. Before ablation, the Si target surface is cleaned ultrasonically in acetone, ethanol, water for $5 \mathrm{~min}$ subsequently. The cleaned $\mathrm{Si}$ wafer $(2 \mathrm{~cm} \times 2 \mathrm{~cm})$ placed in deionized water and the height of liquid layer over the target is adjusted to be $\sim 1 \mathrm{~cm}$. The laser beam is focused on the Si target immersed in deionized water with a spot size about $3.8 \mathrm{~mm}$ in diameter using a $200 \mathrm{~mm}$ focal length. First, ablation was carried out for 200 loops using stationary beam (at a fluence amount of $30 \mathrm{~mJ} \mathrm{~cm}^{-2}$ ) to produce large nanoparticles with wide size distribution (10-100 nm). We will call these reference NPs as yellow Si-NPs. Afterward, bulk target is removed from solution and laser beam with high $\left(60 \mathrm{~mJ} \mathrm{~cm}^{-2}\right)$ and moderate $\left(30 \mathrm{~mJ} \mathrm{~cm}^{-2}\right)$ amount of fluences are applied into the yellow SiNPs solution for 1500 number of spiral scanning to obtain blue and green Si-NPs.

\section{Characterization}

To characterize structural properties of the nanoparticles, transmission electron microscopy (TEM, Tecnai G2-F30, FEI) and high resolution TEM (HRTEM) are performed at $200 \mathrm{kV}$ for Si-NPs dispersed on a holey carbon coated copper grid. Selected area electron diffraction (SAED) pattern of nanoparticles is also collected to understand crystallinity of the prepared nanoparticles. Further investigations on crystallinity and size of nanoparticles are performed using Raman spectroscopy (WITec Alpha 300S scanning near-field optical microscope with Raman module). In order to optically characterize nanoparticles, UVvis-NIR spectrophotometer (Cary 5000, Varian) is employed and photoluminescence (PL) measurements have been performed using Cary Eclipse Fluorescence Spectrophotometer with an excitation wavelength of $310 \mathrm{~nm}$, and the emission spectra are recorded between $400 \mathrm{~nm}$ and $600 \mathrm{~nm}$. Finally, X-ray photoelectron spectroscopy (XPS, Thermoscientic K-Alpha, Al KAlpha radiation, $h v=1486.6 \mathrm{eV}$ ) is utilized to study Si-NPs surface properties and the presence of defects at survey mode by operating flood gun to prevent surface charging with the pass energy and step size set to $30 \mathrm{eV}$ and $0.1 \mathrm{eV}$, respectively.

\section{Results and discussion}

Synthesis route of Si-NPs is schematically depicted at Fig. 1. As shown in Fig. 1a, first, stationary beam is employed for 200 cycles to ablate the Si wafer (target) in deionized water, which gives a widely size distributed Si-NPs (Fig. 1a). We call this reference NPs solution as yellow due to its color appearance. Then bulk target is removed from the solution and spiral beam scanning with two different laser fluences is applied to asprepared NPs to further reduce NPs size (Fig. 1b). Using this spiral scanning scheme, NPs are collected toward the center and get trapped in the active area and laser spot interacts with the particles in every ablation cycle. During every cycle of spiral scanning the ablated nanoparticles are collected toward the center and in the next cycle, they get ablated once again.

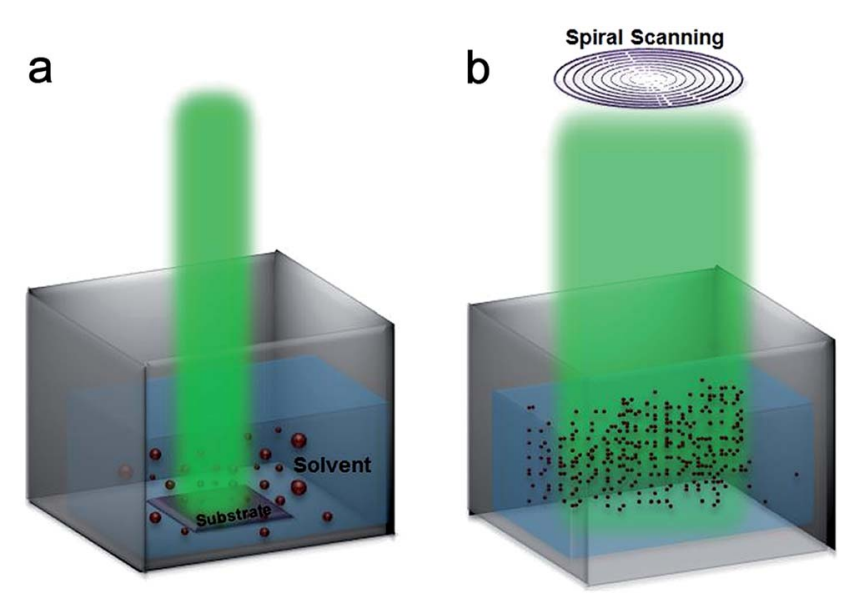

Fig. 1 Schematic representation of NPs synthesis route by (a) stationary beam to produce large Si-NPs and (b) spiral beam to obtain ultra-small Si-NPs ( $3 \mathrm{~nm})$. 
Therefore, resultant Si-NPs can reach to very small sizes in a short time with high uniformity in size distribution.

In this study, we have prepared 2 different NPs samples with high $\left(60 \mathrm{~mJ} \mathrm{~cm}^{-2}\right)$ and moderate $\left(30 \mathrm{~mJ} \mathrm{~cm}^{-2}\right)$ fluences. Both of these samples are obtained after 1500 loops of ablation under spiral scanning scheme. In this paper, these two types of NPs are recalled as blue and green NPs, respectively, due to their emission properties.

TEM images of as-prepared Si-NPs (200 loops) and those of ablated at different fluence levels for large number of loops (1500) are shown at Fig. 2a-f. From these images we can confirm that the obtained Si-NPs by laser ablation (200 loops) are nearly spherical with high size distribution (Fig. 2a). A shown in Fig. 2b, selected area electron diffraction pattern (SAED) of asprepared NPs demonstrates that they are single crystalline, displaying (111) lattice sets with interplanar spacing of $0.31 \mathrm{~nm}$ (characteristic of Si bulk). As shown in Fig. $2 \mathrm{~d}$ and $\mathrm{f}$, this crystalline phase is not kept by green and blue NPs. For green NPs, a polycrystalline feature with several small grain boundaries can be observed while blue Si-NPs are amorphous. Also, as it can be seen from these figures, as-prepared NPs have a broad size distribution from tens to hundreds of $\mathrm{nm}$. However, this distribution has been narrowed to much more uniform sizes with sub $5 \mathrm{~nm}$ dimensions for green (Fig. 2c) and blue (Fig. 2e) nanoparticles. These results are in line with a recent study that has investigated the formation of oxide layer around Si-NP during ablation process. ${ }^{29}$ This paper demonstrates that during laser ablation by nanosecond pulse, two types of morphologies can be formed for the nanocrystals; (1) a polycrystalline $\mathrm{Si}$ core surrounded with a thin $\mathrm{SiO}_{x}$ shell, and (2) a fully oxidized amorphous $\mathrm{SiO}_{x}$. It has been also experimentally demonstrated that the latter case is likely when the temperature is high (above $1050{ }^{\circ} \mathrm{C}$ ). This is in accordance with our findings that higher laser fluences can make higher local heating and initiate full oxidization. Although the exact mechanism of SiNPs ablation in water solution is still debated, this process is generally explained in three phases: $:^{30-32}$ (1) laser induced heating, (2) adiabatic cooling and (3) resultant expansion. When the high intensity laser beam hits the $\mathrm{Si}$, light is absorbed in the $\mathrm{Si}$ target and causes local heating $(\sim 5000 \mathrm{~K})$ where this heat is diffused into the bulk material by conduction, plasma formation and etc. Therefore, in the vicinity of the Si-NPs surface, generated NPs experience surface reconstruction due to laser induced heating and fast cooling. When this process continues for relatively large number of loops, this re-formed NPs get smaller and subsequently several deformations on the Si-NPs surface are created. In the dimensions as small as sub-5 $\mathrm{nm}$, where surface properties start to be dominant over bulk ones, particles have already experienced multiple surface reconstructions. Therefore, the resultant morphology turns to polycrystalline one. Higher levels of fluence will make grain boundaries smaller and eventually the polycrystalline phase will turn to amorphous one as it is the case in blue Si-NPs.

Therefore, depending on the laser pulse fluence, these deformations can make polycrystalline or amorphous NPs. For high levels of laser fluence, in every ablation the grain boundary sizes decrease and finally at dimensions as small as $5 \mathrm{~nm}$ several ablation loops will make NPs to be amorphous.

To have a better comparison, Raman spectroscopy is utilized to evaluate NPs response in higher volumes rather than single nanoparticles (as we analyzed in TEM). Fig. 3 presents Raman responses for both blue and green Si-NPs. As revealed in this figure, both Si-NPs have two characteristic peaks; one is a sharp peak located at $515.8 \mathrm{~cm}^{-1}$ and the other is a broad peak extended toward $450 \mathrm{~cm}^{-1}$ with a weaker amplitude. Sharp peak is representing polycrystalline nature of NPs and the broad one is a sign of amorphous part. Therefore, by comparing these two peaks, we can find that the polycrystalline nature is dominant for green NPs while for that of blue NPs, amorphous portion is comparable with polycrystalline peak. Another feature that should be considered is the position of the polycrystalline peak which is downshifted by $-4.2 \mathrm{~cm}^{-1}$ compared to reference bulk
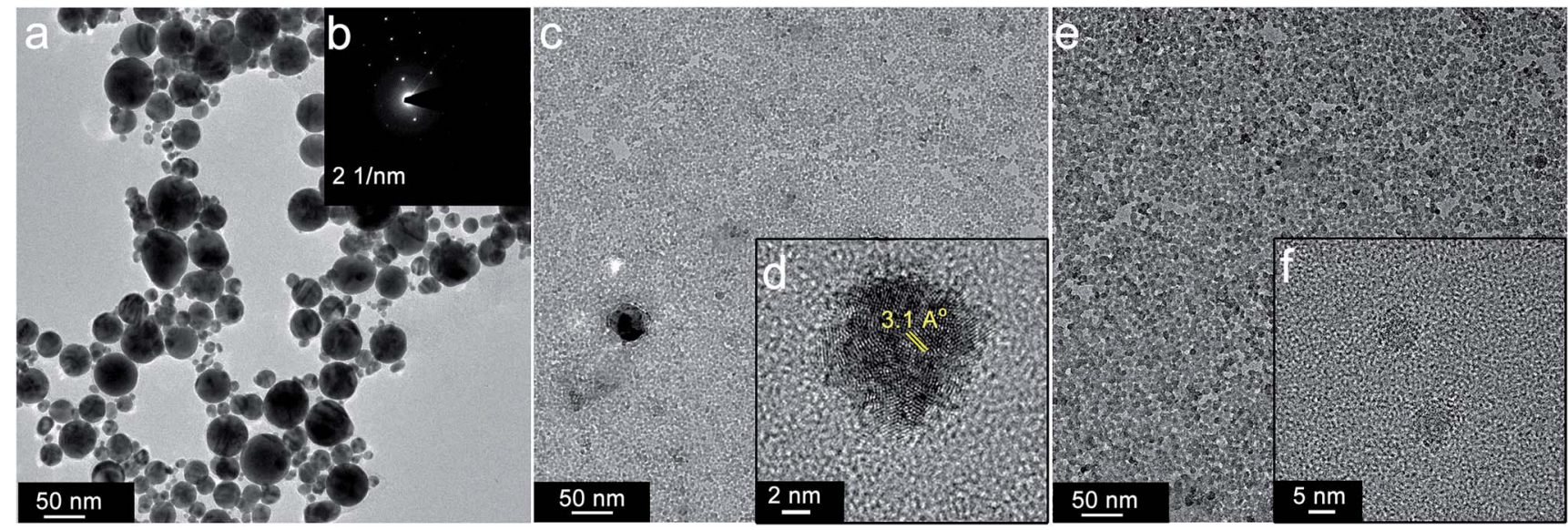

Fig. 2 TEM images of Si-NPs obtained by nanosecond laser ablation prepared at different laser fluences; (a) as-prepared yellow Si-NPs and (b) their SAED pattern, (c) under $30 \mathrm{~mJ} \mathrm{~cm}^{-2}$ laser fluence ablated green Si-NPs and (d) its HRTEM image of individual Si-NP with high magnification interface, indicating 3.1 A lattice fringes, and (e) $60 \mathrm{~mJ} \mathrm{~cm}^{-2}$ laser fluence used to synthesize blue Si-NPs and (f) its HRTEM image showing the amorphous nature of the resultant nanoparticles. 


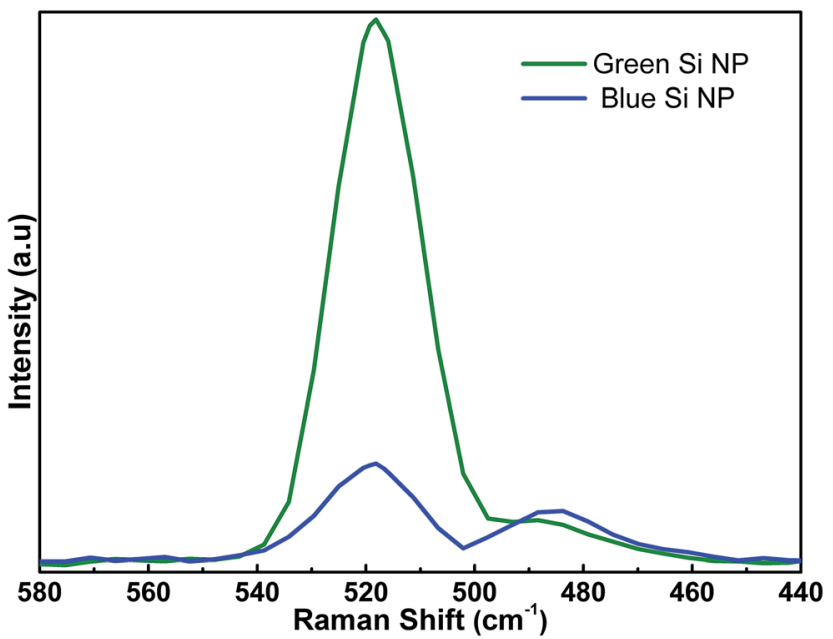

Fig. 3 Raman spectra of the blue and green Si-NPs.

Si $\left(520 \mathrm{~cm}^{-1}\right)$. This shift proves the existence of quantum confined Si-NPs; the dependence of this peak shift $(\Delta \omega)$ with SiNPs size $(D)$ can be explained by: ${ }^{33}$

$$
\Delta \omega=-52.3(0.543 / D)^{1.586}
$$

Evaluating this equation with our measured values, we conclude that both blue and green NPs have the same estimated mean size of $c a .2 .8 \mathrm{~nm}$. Further investigations on NPs size and their optical response is conducted using UV-Vis-NIR spectroscopy, Fig. 4 represents absorption spectra of different sized SiNPs. As it can be seen, larger sized Si-NPs (yellow ones) show an absorption edge in the near infrared (NIR) region which is inherent absorption of the bulk Si. This is expected considering the fact that generated Si-NPs have relatively large diameters (compared to $\sim 4 \mathrm{~nm}$ exciton Bohr radius of Si). However, the absorption edge of Si-NPs gets blue shifted due to quantum confinement effect. It is well known that when the sizes When

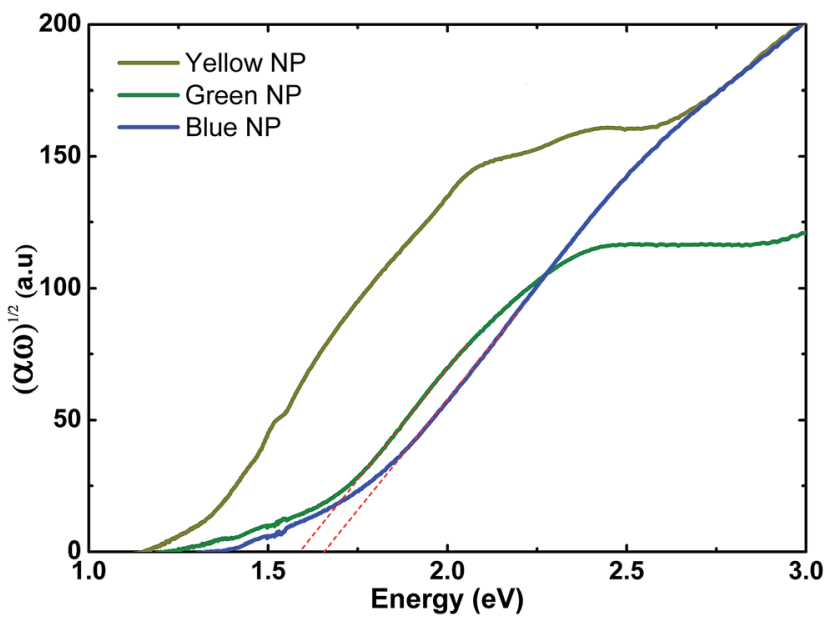

Fig. 4 Optical absorption edge, $(\alpha \omega)^{1 / 2}$ as a function of photon energy $(h \nu)$, measured from the nanoparticles; named with yellow, green and blue. dimensions of an indirect semiconductor approaches to dimensions as small as its exciton Bohr radius, it turns to a direct band gap semiconductor and experiences band gap widening. In our case, the band gap of NPs is calculated by extrapolation of the linear part of the $(\alpha \omega)^{1 / 2}$ versus $h \nu$ axis in the wavelength range of 1-3 $\mu \mathrm{m}$. These findings show that the corresponding band gaps for green and blue NPs are $1.59 \mathrm{eV}$ and $1.64 \mathrm{eV}$, respectively. According to previous studies, the size of Si-NPs (with dimensions of $2-10 \mathrm{~nm}$ ) can be estimated using below formula; ${ }^{34}$

$$
E_{\mathrm{g}}=E_{0}+A \times D^{-2}+B \times D^{-1}
$$

where $E_{0}$ is the band gap of bulk $\mathrm{Si}(1.12 \mathrm{eV})$, and $D$ is diameter of the NP. Also two constants of $A=2.56 \mathrm{eV} \mathrm{nm}^{-2}$ and $B=$ $0.83 \mathrm{eV} \mathrm{nm}^{-2}$ are experimentally obtained from fitting results. According to this model, the average size of blue and green NPs is estimated to be $3.2 \mathrm{~nm}$ and $3.4 \mathrm{~nm}$, respectively. This is in good agreement with our previous results where we estimated NPs size to be almost same in a mean amount of $2.8 \mathrm{~nm}$. All of these results show that the fabricated nanoparticles have the same size regardless of laser fluence. The reason is our spiral line beam scanning scheme that captures NPs in the active area and ablate them in each cycle as previously stated. Therefore, regardless of the laser fluence, NPs will have the same size at the end of the ablation process.

After all of these investigations that revealed both green and blue NPs have same size, photoluminescence (PL) spectroscopy is utilized to explore emission properties of Si-NPs. For this aim, PL spectra of Si-NPs under an excitation wavelength of $310 \mathrm{~nm}$ are recorded for both green and blue NPs. As it can be seen from Fig. 5, the emission for both of NPs is comprised of two main peaks located at $426 \mathrm{~nm}$ (blue emission) and $520 \mathrm{~nm}$ (green emission). However, the strength of these peaks is different for two solutions.

To have a better comparison, the ratio of PL ( $426 \mathrm{~nm}$ )/PL (520 $\mathrm{nm}$ ) is calculated. Based on these results this ratio for blue

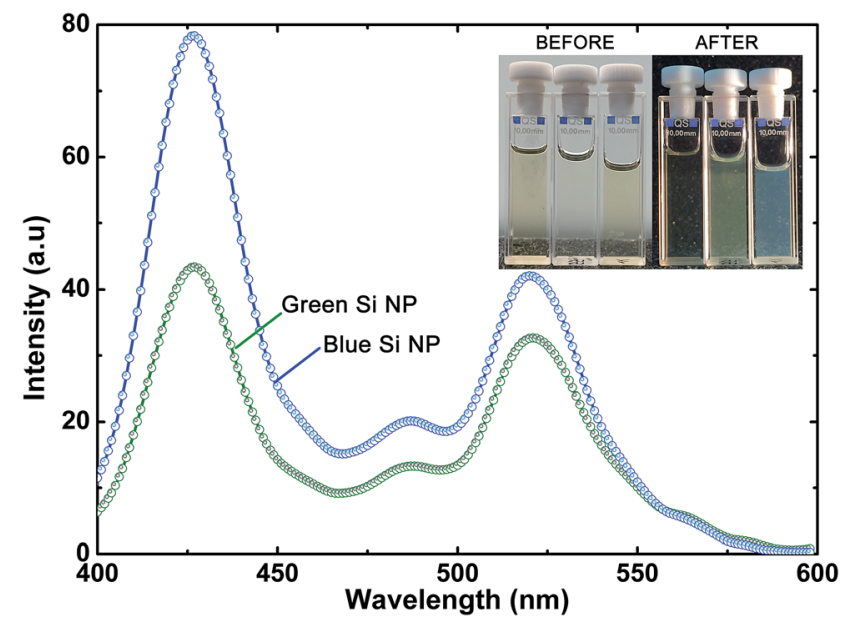

Fig. 5 PL spectra of blue and green Si-NPs excited at a wavelength of $310 \mathrm{~nm}$. Inset shows intense visible (near red) luminescence under halogen lamp NPs before and after excitation. 
emitting solution is about $\sim 1.85$, while this amount is 1.33 for green one. The inset of the figure shows NPs before and after excitation with a halogen lamp (white light source with UV component). As indicated before, $\mathrm{Si}$, as its bulk is an indirect band gap semiconductor with very weak emission property. However, when its dimensions shrink down, due to quantum confinement, the probability of direct transition will increase and consequently its emission yield is intensified. Thus, in our case, this emission could be a band-to-band transition as a consequence of quantum confinement effect. Also, due to existence of Si-NPs with different sizes, the emission should be tuned upon change in the excitation wavelength. So we repeated PL experiment with three different excitations; $290 \mathrm{~nm}, 310 \mathrm{~nm}$, and $330 \mathrm{~nm}$. As it can be clearly seen from Fig. 6a and b, moving toward higher excitation wavelengths for both solutions the blue emission at $426 \mathrm{~nm}$ gets a red shift which confirms the existence of quantum confinement effect in the solutions. Also, peak broadening in higher wavelength excited samples can originates from inhomogeneous broadening due to highly distributed defect states inside $\mathrm{SiO}_{x}$ band gap. ${ }^{35}$ It has been shown that several defect states at different energetic locations are existed inside $\mathrm{SiO}_{x}$ layer. According to the excitation wavelength, these states can be activated and even dominate the
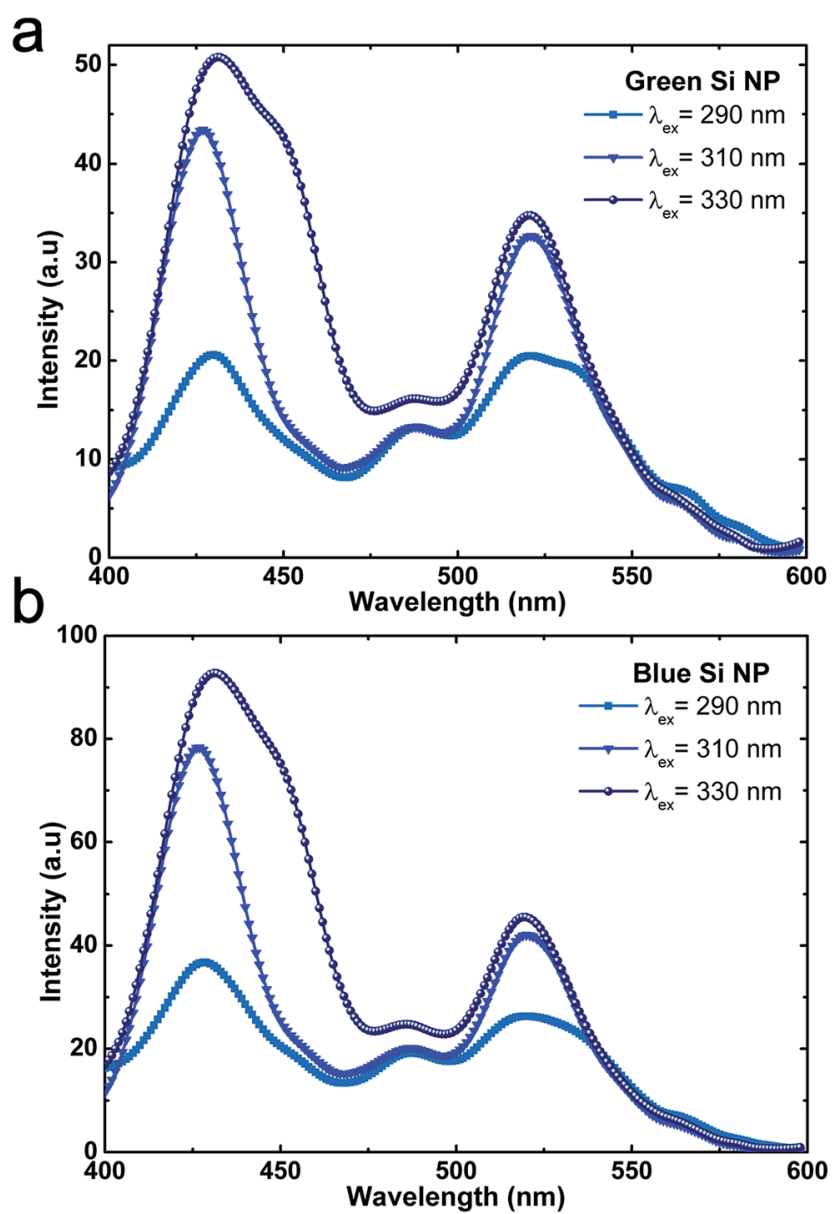

Fig. 6 PL spectra of (a) green and (b) blue Si-NPs with different excitation wavelengths. emission properties of the layer. It should be noted that the peak at $520 \mathrm{~nm}$ does not exhibit any shift which implies that these two peaks do not arise from the same origin. To have a better understanding on the origin of these two peaks, the impact of post-treatment by hydrofluoric acid (HF) on the PL emission of NPs is examined. For this aim, 5\% HF is added to both blue and green emitting silicon NPs solution and PL emission of NPs is recorded in every 1 min intervals. Fig. 7a and $\mathrm{b}$ reveals that for both cases the blue peak $(426 \mathrm{~nm})$ is quenched after HF post-treatment while the green peak is almost unchanged. However, this quench is much more pronounced in the case of blue emitting NPs. Considering the fact that HF removes the $\mathrm{SiO}_{x}$ layer by chemical etching, the reduction in the first peak ( $426 \mathrm{~nm}$ ) can show us that this peak is mainly due to oxide related defects at $\mathrm{Si} / \mathrm{SiO}_{x}$ interface. This conclusion is in agreement with some of the previous studies where the blue emission is contributed to the defects at $\mathrm{Si} / \mathrm{SiO}_{x}$ interface ${ }^{29,36}$ rather than direct recombination in ultra-small $(<5 \mathrm{~nm}) \mathrm{Si}$ nanocrystals (due to quantum confinement). These studies show that the blue emission from Si nanocrystals is occurred at energy of $2.7 \mathrm{eV}$ and this is mainly due to a forbidden triplet-tosinglet transition inside the $\mathrm{SiO}_{x}$ shell layer. On the other side,
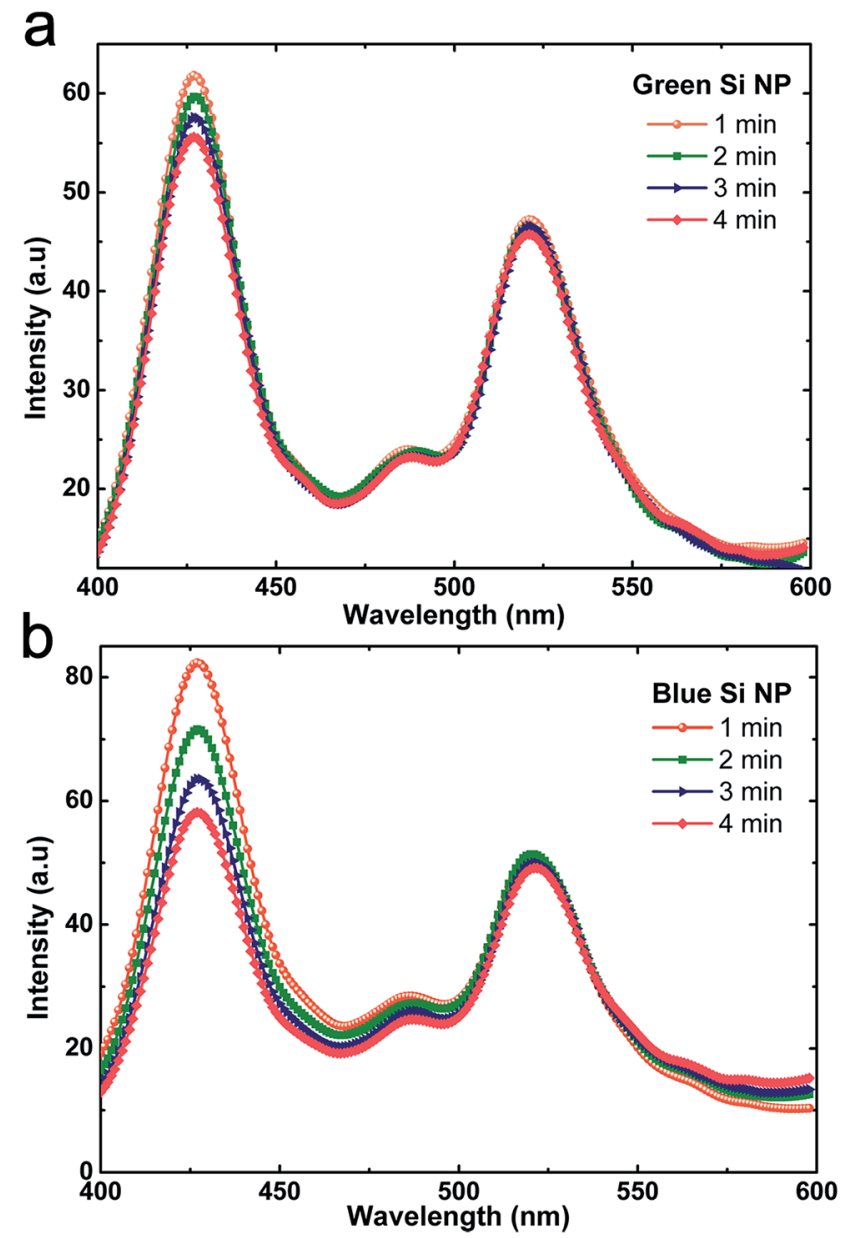

Fig. 7 The impact of hydrofluoric acid post-treatment on PL spectra of (a) green and (b) blue emitting Si-NPs. 
a

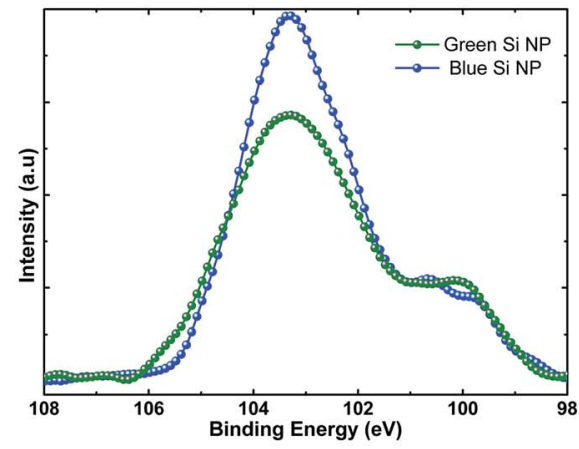

b

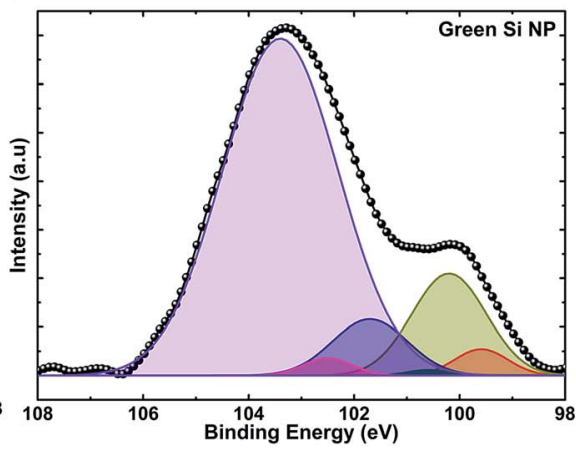

C

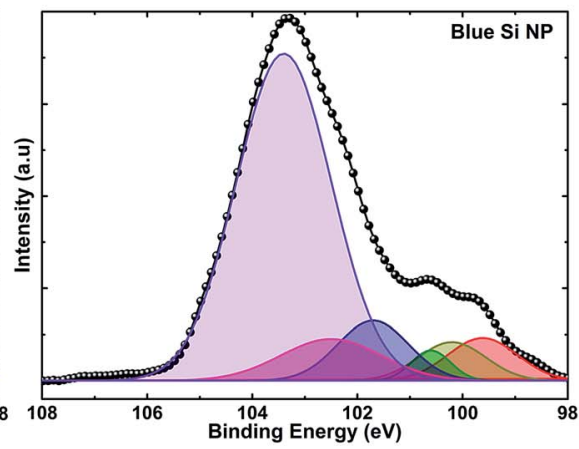

Fig. 8 Si2p XPS spectrum of (a) green and blue Si-NPs and ( $b$ and c) their corresponding fitting. The Gaussian peaks belong to; Si2p $3 / 2$ at 99.6 eV (red), $\mathrm{Si}_{2} \mathrm{p}_{1 / 2}$ at $100.2 \mathrm{eV}$ (dark yellow), $\mathrm{Si}_{2} \mathrm{O}$ at $100.6 \mathrm{eV}$ (Olive), $\mathrm{SiO}$ at $101.7 \mathrm{eV}$ (navy), $\mathrm{Si}_{2} \mathrm{O}_{3}$ at $102.5 \mathrm{eV}$ (pink), $\mathrm{SiO}_{2}$ at $103.4 \mathrm{eV}$ (violet).

the lack of any change for green peak $(520 \mathrm{~nm})$ represents that this emission stems from non-oxide related defects that are mainly grain boundaries at NPs surface. This fact can also be confirmed by comparing the decline strength for green and blue emitting NPs. The abrupt reduction for the case of blue emitting NPs reveals that the oxide related defects are more pronounced compared to that of green NPs solution. This can also be confirmed by reviewing the laser ablation process. When the level of laser fluence exceeds some threshold level, oxide layer formation can be initiated during the NPs formation.

To have an insight on density and nature of these oxide defects, XPS measurement is also carried out for both solutions. Fig. 8a compares Si2p spectra for two different solutions. These spectra have been deconvoluted to 6 different Gaussian functions; (1) Si peaks ( $\mathrm{Si} 2 \mathrm{p}_{3 / 2}$ at $99.6 \mathrm{eV}, \mathrm{Si}_{2} \mathrm{p}_{1 / 2}$ at $100.2 \mathrm{eV}$ ) and $\mathrm{SiO}_{x}$ peaks $\left(\mathrm{Si}_{2} \mathrm{O}\right.$ at $100.6 \mathrm{eV}, \mathrm{SiO}$ at $101.7 \mathrm{eV}, \mathrm{Si}_{2} \mathrm{O}_{3}$ at $102.5 \mathrm{eV}$, $\mathrm{SiO}_{2}$ at $103.4 \mathrm{eV}$ ). As shown in Fig. $8 \mathrm{~b}$ and c, the percentage portion of area under $\mathrm{SiO}_{x}$ peaks is $85 \%$ and $74 \%$ for blue and green emitting Si-NPs, respectively. This confirms our hypothesis that the $\mathrm{SiO}_{x}$ layer formation is more likely under higher levels of laser fluence. On the other hand, the portion of nonstoichiometric $\mathrm{SiO}_{x}(x \neq 2)$ part is $25 \%$ for blue emitting $\mathrm{Si}$ NPs while this amount is $14 \%$ for green ones. This demonstrates that, for blue emitting Si-NPs, density of oxide related defects at $\mathrm{Si} / \mathrm{SiO}_{x}$ interface is higher compared to that of green NPs.

\section{Conclusions}

In this work, we conducted a systematic analysis on the origin of green and blue emissions from Si-NPs synthesized directly into the water by employing nanosecond pulsed laser ablation of Si bulk target. For this aim, first, we introduced a new ablation methodology based on spiral line scanning that ensures formation of ultra-small (as small as $\sim 3 \mathrm{~nm}$ ), highly uniform luminescent NPs. Since NPs get trapped in the active area using this scanning scheme, laser spot interacts and ablates them during every cycle of scanning. Thus, in a short time, very small size Si-NPs with highly uniformity can be attained. Furthermore, It was demonstrated that by controlling the laser fluence, the luminescense of Si-NPs can be tuned between blue and green. Although both types of Si-NPs had almost same dimensions, their crystallinity was different in which Si-NPs synthesized at $30 \mathrm{~mJ} \mathrm{~cm}^{-2}$ show polycrystalline nature while the ones ablated at $60 \mathrm{~mJ} \mathrm{~cm}{ }^{-2}$ were amorphous. This difference can cause emission of Si-NPs to be tuned between blue and green. We found that the blue emission (at $426 \mathrm{~nm}$ ) is dominated with defect states at $\mathrm{Si} / \mathrm{SiO}_{x}$ interface, while that of green one (at 520 $\mathrm{nm}$ ) is originated from disorders at the grain boundaries of the polycrystalline Si-NPs. At the high levels of laser fluence, high local heating makes adiabatic melting/cooling and subsequently grain boundary sizes of polycrystalline Si get smaller. For large number of laser ablation cycles, material experiences multiple surface reconstructions which turns its polycrystalline nature to amorphous one as it is the case for blue emitting SiNPs. Our findings and the proposed method can be expanded to other type of materials where tunable structural and optical properties can be attained by adjusting the laser power.

\section{Acknowledgements}

This work was partially supported by the Scientific and Technological Research Council of Turkey (TUBITAK) under Grant Nos 112M004, 112E052, and 113M815. A. K. O. acknowledges support from the Turkish Academy of Sciences Distinguished Young Scientist Award (TUBA GEBIP), BAGEP Award and FABED Award.

\section{References}

1 H. Chang and S.-Q. Sun, Chin. Phys. B, 2014, 23, 88102.

2 J. G. C. Veinot, Chem. Commun., 2006, 4160-4168.

3 A. S. Heintz, M. J. Fink and B. S. Mitchell, Adv. Mater., 2007, 19, 3984-3988.

4 W. A. Su and W. Z. Shen, Appl. Phys. Lett., 2012, 100, 071111.

5 R. Koole, E. Groeneveld and D. Vanmaekelbergh, Nanoparticles, 2014, 13-52.

6 Z. T. Kang, B. Arnold, C. J. Summers and B. K. Wagner, Nanotechnology, 2006, 17, 4477-4482. 
7 H. Morisaki, F. W. Ping, H. Ono and K. Yazawa, J. Appl. Phys., 1991, 70, 1869-1870.

8 H. Tamura, M. Rückschloss, T. Wirschem and S. Vepřek, Appl. Phys. Lett., 1994, 65, 1537-1539.

9 C. M. Gonzalez and J. G. C. Veinot, J. Mater. Chem. C, 2016, 4, 4836-4846.

10 E. J. Henderson, A. J. Shuhendler, P. Prasad, V. Baumann, F. Maier-Flaig, D. O. Faulkner, U. Lemmer, X. Y. Wu and G. A. Ozin, Small, 2011, 7, 2507-2516.

11 R. K. Baldwin, K. A. Pettigrew, E. Ratai, M. P. Augustine and S. M. Kauzlarich, Chem. Commun., 2002, 1822-1823.

12 J. R. Heath, Science, 1992, 258, 1131-1133.

13 J. Zou, P. Sanelle, K. A. Pettigrew and S. M. Kauzlarich, J. Cluster Sci., 2006, 17, 565-578.

14 R. A. Bley and S. M. Kauzlarich, J. Am. Chem. Soc., 1996, 118, 12461-12462.

15 M. H. Nayfeh, N. Barry, J. Therrien, O. Akcakir, E. Gratton and G. Belomoin, Appl. Phys. Lett., 2001, 78, 1131-1133.

16 O. Akcakir, J. Therrien, G. Belomoin, N. Barry, J. D. Muller, E. Gratton and M. Nayfeh, Appl. Phys. Lett., 2000, 76, 1857.

17 L. Mangolini, E. Thimsen and U. Kortshagen, Nano Lett., 2005, 5, 655-659.

18 H. Takagi, H. Ogawa, Y. Yamazaki, A. Ishizaki and T. Nakagiri, Appl. Phys. Lett., 1990, 56, 2379-2380.

19 G. Ledoux, O. Guillois, D. Porterat, C. Reynaud, F. Huisken, B. Kohn and V. Paillard, Phys. Rev. B: Condens. Matter Mater. Phys., 2000, 62, 15942-15951.

20 M. Ehbrecht, B. Kohn, F. Huisken, M. Laguna and V. Paillard, Phys. Rev. B: Condens. Matter Mater. Phys., 1997, 56, 6958-6964.

21 P. Chewchinda and T. Tsuge, Jpn. J. Appl. Phys., 2013, 52, 120699.

22 A. Hamad, L. Li and Z. Liu, Appl. Phys. A, 2015, 120, 12471260.
23 K. Abderrafi, R. G. Calzada, M. B. Gongalsky, I. Suarez, R. Abarques, V. S. Chirvony, V. Y. Timoshenko, R. Ibanez and J. P. Martinez-Pastor, J. Phys. Chem., 2011, 115, 51475151.

24 L. Vaccaro, P. Camarda, F. Messina, G. Buscarino, S. Agnello, F. M. Gelardi, M. Cannas and R. Boscaino, AIP Conf. Proc., 2014, 1624, 1-6.

25 S. Alkis, A. K. Okyay and B. Ortaç, J. Phys. Chem. C, 2012, 116, 3432-3436.

26 X. D. Pi, R. W. Liptak, J. Deneen Nowak, N. P. Wells, C. B. Carter, S. A. Campbell and U. Kortshagen, Nanotechnology, 2008, 19, 245603.

27 S. Yang, W. Li, B. Cao, H. Zeng and W. Cai, J. Phys. Chem. C, 2011, 115, 21056-21062.

28 I. D. Rukhlenko, M. Y. Leonov, V. K. Turkov, A. P. Litvin, A. S. Baimuratov, A. V. Baranov and A. V. Fedorov, Opt. Express, 2012, 20, 27612-27635.

29 L. Vaccaro, R. Popescu, F. Messina, P. Camarda, R. Schneider, D. Gerthsen, F. M. Gelardi and M. Cannas, J. Appl. Phys., 2016, 120, 024303.

30 V. Amendola and M. Meneghetti, Phys. Chem. Chem. Phys., 2013, 15, 3027-3046.

31 Z. Yan and D. B. Chrisey, J. Photochem. Photobiol., C, 2012, 13, 204-223.

32 H. Zeng, X. W. Du, S. C. Singh, S. A. Kulinich, S. Yang, J. He and W. Cai, Adv. Funct. Mater., 2012, 22, 1333-1353.

33 S. Yang, W. Cai, H. Zhang, X. Xu and H. Zeng, J. Phys. Chem. C, 2009, 113, 19091-19095.

34 X. Liu, Y. Zhang, T. Yu, X. Qiao, R. Gresback, X. Pi and D. Yang, Part. Part. Syst. Charact., 2016, 33, 44-52.

35 L. Skuja, J. Non-Cryst. Solids, 1998, 239, 16-48.

36 L. Vaccaro, L. Sciortino, F. Messina, G. Buscarino, S. Agnello and M. Cannas, Appl. Surf. Sci., 2014, 302, 62-65. 